Angew Chem Int Ed Engl. 2017 May 15; 56(21): 5821-5824. doi:10.1002/anie.201702402.

\title{
Control of Vicinal Stereocenters via Nickel-Catalyzed Alkyl-Alkyl Cross-Coupling
}

Xin Mu, Yu Shibata, Yusuke Makida, and Prof. Gregory C. Fu

Division of Chemistry and Chemical Engineering, California Institute of Technology, Pasadena, CA, 91125 (USA)

\section{Abstract}

Vicinal stereocenters are found in many natural and unnatural compounds. Although metalcatalyzed cross-coupling reactions of unactivated alkyl electrophiles are emerging as a powerful tool in organic synthesis, there have been virtually no reports of processes that generate, much less control, vicinal stereocenters. In this investigation, we establish that a chiral nickel catalyst can achieve doubly stereoconvergent alkyl-alkyl cross-couplings, specifically, reactions of a racemic pyrrolidine-derived nucleophile with cyclic alkyl halides (as mixtures of stereoisomers) to produce vicinal stereocenters with very good stereoselectivity.

\section{Table of Contents}

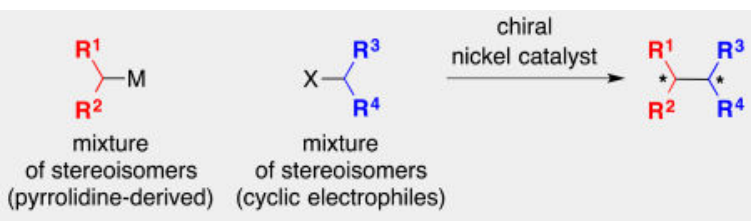

In this investigation, we establish that a chiral nickel catalyst can achieve doubly stereoconvergent alkyl-alkyl cross-couplings, specifically, reactions of a racemic pyrrolidine-derived nucleophile with cyclic alkyl halides (as mixtures of stereoisomers) to produce vicinal stereocenters with very good stereoselectivity.

\section{Keywords}

alkylation; asymmetric synthesis; cross-coupling; nickel; zinc

The transition-metal-catalyzed cross-coupling of an alkyl nucleophile with an unactivated alkyl electrophile to generate an alkyl-alkyl bond is emerging as a powerful tool for the synthesis of organic molecules. ${ }^{1}$ For many such processes, the challenge is two-fold: constructing the desired carbon-carbon bond and controlling the stereochemistry at one or both carbons. Stereoconvergent reactions, wherein a catalyst converts a mixture of stereoisomeric substrates into a particular stereoisomer of the product, are attractive, since such substrate mixtures are readily available (Figure 1). A number of methods for 
stereoconvergent metal-catalyzed alkyl-alkyl cross-coupling have been described that control stereochemistry at one, but not both, of the carbons of the new carbon-carbon bond (top of Figure 1). ${ }^{1,2,3}$ In contrast, there are no examples of effective control of vicinal stereocenters in cross-couplings of unactivated secondary electrophiles, due to the difficulty of achieving bond formation, ${ }^{4}$ much less stereoselectivity (maximum $\left.\mathrm{dr}:<1.5: 1\right){ }_{.}^{4 \mathrm{~d}}$

Because pyrrolidine subunits are present in many natural and non-natural compounds, ${ }^{5} \mathrm{a}$ variety of methods for the asymmetric synthesis of chiral pyrrolidines have been described, including catalytic enantioselective processes. ${ }^{6}$ For example, we have recently reported a nickel-catalyzed enantioconvergent cross-coupling of racemic ${ }^{7}$ a-zincated N-Bocpyrrolidine with an array of achiral secondary alkyl electrophiles [Eq. (1)]. ${ }^{2}$

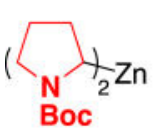

0.75 equiv

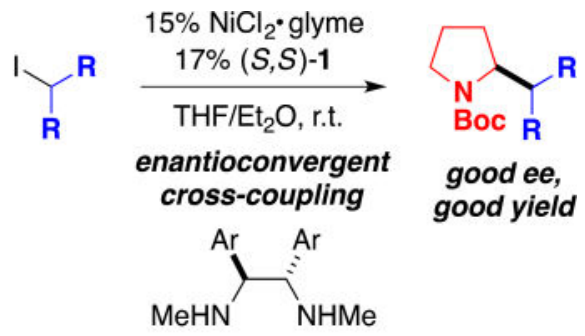

$(S, S)-1$

$\mathrm{Ar}=1$-naphthyl

We decided to pursue the possibility that this method could provide control of vicinal stereocenters in alkyl-alkyl couplings. Specifically, we examined the cross-coupling of 2zincated $N$-Boc-pyrrolidine with a 4 -substituted cyclohexyl halide ${ }^{8}$ (entry 1 of Table 1 ). Under our previously reported conditions, ${ }^{2}$ we generated the coupling product with very good stereoselectivity ( $92 \%$ ee, $91: 9 \mathrm{dr}$ ). Through the use of a different nickel source $\left(\mathrm{Ni}(\mathrm{acac})_{2}\right)$, we were able to obtain similar results while employing a smaller excess of the nucleophile ( 0.6 instead of 0.75 equiv of $\left.\mathrm{ZnR}_{2}\right) .{ }^{9}$ The ee and the $\mathrm{dr}$ of the cross-coupling product remain constant throughout the course of the reaction.

This method achieves good control of vicinal ${ }^{10}$ stereocenters in alkyl-alkyl cross-couplings of an array of achiral cyclohexyl and tetrahydropyranyl iodides (Table 1). For example, Negishi reactions of 4-monosubstituted coupling partners proceed with very good ee and diastereoselectivity with a sterically and electronically diverse set of substituents (Me, Et, $t$ $\mathrm{Bu}, \mathrm{CF}_{3}$, and $\mathrm{Ph}$; Table 1, entries 1-5); except in the case of the methyl-substituted electrophile, essentially one diastereomer is observed (>99:1). Furthermore, the alkyl-alkyl cross-coupling of a 4,4-disubstituted cyclohexyl iodide occurs with good stereoselectivity (entry 6). Finally, excellent control of vicinal stereocenters is observed in Negishi reactions of 3,5-disubstituted electrophiles, including an oxygen heterocycle (entries 7 and 8).

Because high stereoselectivity and high yield can be obtained in the cross-coupling of a racemic nucleophile (i.e., prepared from racemic 2 -lithio- $N$-Boc-pyrroline) with an electrophile that is an $\sim 2: 1$ mixture of stereoisomers (entries 4 and 7 of Table 1), these Negishi reactions are doubly stereoconvergent (bottom of Figure 1). In the case of the 
stereogenic center on the pyrrolidine ring, the absolute stereochemistry is controlled by the chiral catalyst. ${ }^{2}$ The stereochemistry at the other carbon of the new carbon-carbon bond is dependent upon the structure of the electrophile. We hypothesize that the nickel catalyst abstracts iodine from the electrophile, ${ }^{11}$ which generates the same alkyl radical from either diastereomer of the alkyl iodide. In all cases in Table 1, the major stereoisomer of the product bears the new alkyl substituent in the thermodynamically favored equatorial position on the six-membered ring; this may be due to a kinetic preference during the reaction of the alkyl radical with nickel to form a nickel-carbon bond, ${ }^{12}$ or to reversible nickel-carbon bond formation and stereochemistry-determining reductive elimination. ${ }^{13}$

By conducting the asymmetric cross-coupling with a single diastereomer of the alkyl iodide and stopping the reaction at partial conversion, we have determined that the electrophile undergoes isomerization during the reaction [Eq. (1) and Eq. (2)]; in each case, the crosscoupling product is generated in $92 \%$ ee and with $>99: 1 \mathrm{dr}$ ). Possible mechanisms for this isomerization include halogen exchange via an $\mathrm{S}_{\mathrm{N}} 2$ reaction (e.g., by zinc iodide that is generated during the cross-coupling) and reversible transfer of $\mathrm{I} \bullet$ from the alkyl iodide to the nickel catalyst. Because the two isomers of the electrophile do not interconvert in the presence of an iodide source ( 1 equiv $\mathrm{ZnI}_{2}$ or TBAI; THF/Et $2 \mathrm{O}$, r.t., $48 \mathrm{~h}$ ), we favor reversible iodine transfer between the alkyl radical and nickel as the more likely pathway. ${ }^{14}$ Because the dr's of the alkyl iodides at partial conversion are different in [Eq. (2)] versus [Eq. (3)], this reversible iodine transfer is not occurring extremely rapidly relative to crosscoupling.
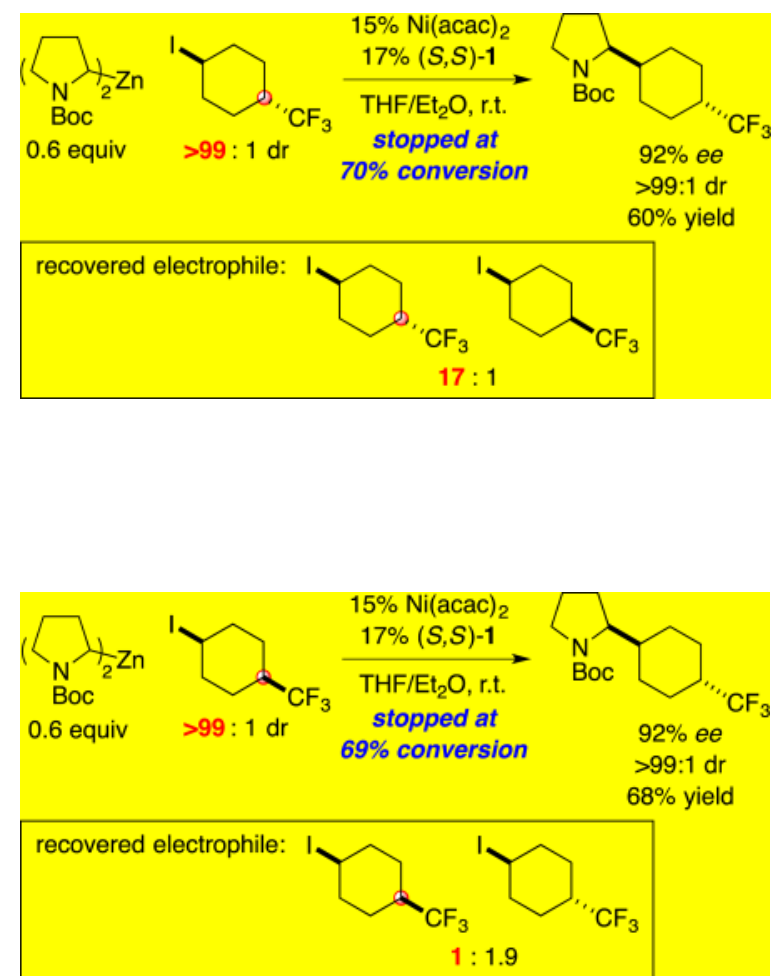
Not only achiral, but also chiral, electrophiles serve as suitable substrates for these nickelcatalyzed stereoselective alkyl-alkyl cross-couplings, generating vicinal stereocenters with good selectivity (Table 2). ${ }^{15}$ Thus, a 3-substituted carbocyclic or heterocyclic alkyl iodide reacts with high stereoselectivity to form the cis-1,3-disubstituted product (entries 1-3). Furthermore, coupling of a 3,3-disubstituted electrophile proceeds with good stereoselection (entry 4). Finally, bicyclic and polycyclic secondary alkyl iodides can be employed as crosscoupling partners (entries 5 and 6).

When the $(R, R)$, rather than the $(S, S)$, enantiomer of ligand $\mathbf{1}$ is used, similar stereoselectivity is generally observed, with the major products differing only in the stereochemistry at the position a to nitrogen (Table 1). This is consistent with control of that stereocenter by the chiral catalyst and control of the second stereocenter by the substrate.

In summary, alkyl-alkyl cross-couplings have now been expanded to include an array of processes wherein vicinal stereocenters, rather than a single stereocenter, are controlled with high selectivity. In particular, a nickel/diamine catalyst accomplishes doubly stereoconvergent Negishi couplings of an alkylzinc reagent with an alkyl halide; with respect to the new carbon-carbon bond, the chiral catalyst controls the stereochemistry of the carbon that originates from the nucleophile, and the substrate controls the stereochemistry of the carbon that originates from the electrophile. Given the frequency with which vicinal stereocenters are encountered in organic molecules, we anticipate that the development of methods that achieve doubly stereoconvergent alkyl-alkyl bond formation will emerge as a highly active area of research in the coming years.

\section{Supplementary Material}

Refer to Web version on PubMed Central for supplementary material.

\section{Acknowledgments}

Support has been provided by the National Institutes of Health (National Institute of General Medical Sciences: R01-GM62871), the Shanghai Institute of Organic Chemistry (fellowship to XM), and the Japan Society for the Promotion of Science (fellowship to YM). We thank Jun Myun Ahn (x-ray crystallography), Lawrence M. Henling (Caltech X-Ray Crystallography Facility), Dr. Nathan Schley (x-ray crystallography), Samantha E. Shockley (Stoltz lab), Dr. Scott C. Virgil (Caltech Center for Catalysis and Chemical Synthesis), Dr. David G. VanderVelde (Caltech NMR facility), and Dr. Haolin Yin for assistance.

\section{References}

1. Choi J, Fu GC. Science. accepted for publication.

2. For use of a racemic nucleophile, see: Cordier CJ, Lundgren RJ, Fu GC. J. Am. Chem. Soc. 2013; 135:10946-10949. [PubMed: 23869442] We speculate that enantioconvergence is achieved through reversible homolysis of the nickel-pyrrolidinyl bond.

3. For use of a racemic electrophile, see: Saito B, Fu GC. J. Am. Chem. Soc. 2008; 130:6694-6695. [PubMed: 18447357] Schmidt J, Choi J, Liu AT, Slusarczyk M, Fu GC. Science. 2016; 354:12651269. [PubMed: 27940868]

4. Hohn E, Pietruszka J. Adv. Synth. Catal. 2004; 346:863-866.(one example: cyclopropylboron reagent with cyclopropyl electrophile); Ren P, Vechorkin O, von Allmen K, Scopelliti R, Hu X. J. Am. Chem. Soc. 2011; 133:7084-7095. [PubMed: 21500782] (one example: 7\% yield); Zultanski SL, Fu GC. J. Am. Chem. Soc. 2011; 133:15362-15364. [PubMed: 21913638] (one example: cyclopropylboron reagent); Yang C-T, Zhang Z-Q, Liang J, Liu J-H, Lu X-Y, Chen H-H, Liu L. J. 
Am. Chem. Soc. 2012; 134:11124-11127. [PubMed: 22734716] (Grignard reagents as nucleophiles); e) Reference 2 (nucleophile: a-zincated $N$-Boc-pyrrolidine).

5. For examples and leading references, see: a) Table 6 in: Roughley SD, Jordan AM. J. Med. Chem. 2011; 54:3451-3479. [PubMed: 21504168] Michael JP. Nat. Prod. Rep. 2008; 25:139-165. [PubMed: 18250900] Michael JP. Alkaloids. 2001; 55:91-258. [PubMed: 11704985] Rizk AFM. Naturally Occurring Pyrrolizidine Alkaloids. CRC PressBoca Raton1991

6. For example, see: Brown AR, Uyeda C, Brotherton CA, Jacobsen EN. J. Am. Chem. Soc. 2013; 135:6747-6749. [PubMed: 23597402] Trost BM, Lam TM, Herbage MA. J. Am. Chem. Soc. 2013; 135:2459-2461. [PubMed: 23363050]

7. The organozinc reagent is prepared via lithiation with $s$-BuLi (no chiral additive), generating a racemic organolithium reagent $(\mathrm{RLi})$. Reaction with $\mathrm{ZnI}_{2}$ affords a diorganozinc reagent $\left(\mathrm{ZnR}_{2}\right)$ that may bear homochiral or heterochiral substituents (R).

8. For systematic studies of the use of an achiral catalyst to achieve diastereoselective cross-couplings of primary alkyl nucleophiles with cyclic alkyl electrophiles, see: Perez Garcia PM, Di Franco T, Orsino A, Ren P, Hu X. Org. Lett. 2012; 14:4286-4289. [PubMed: 22849761] Gong H, Sinisi R, Gagné MR. J. Am. Chem. Soc. 2007; 129:1908-1909. [PubMed: 17261000]

9. Use of a lower catalyst loading $\left(10 \% \mathrm{Ni}(\mathrm{acac})_{2} / 12 \%(S, S)-\mathbf{1}\right)$ led to formation of the product in $92 \%$ ee, $93: 7 \mathrm{dr}$, and $85 \%$ yield.

10. According to the IUPAC Compendium of Chemical Terminology (https://goldbook.iupac.org/ S05980.html; accessed 21 February 2017), a stereogenic center is a "grouping of atoms consisting of a central atom and distinguishable ligands, such that the interchange of any two of the substituents leads to a stereoisomer."

11. a) Zhou J, Fu GC. J. Am. Chem. Soc. 2004; 126:1340-1341. [PubMed: 14759182] b) Anderson TJ, Jones GD, Vicic DA. J. Am. Chem. Soc. 2004; 126:8100-8101. [PubMed: 15225035] c) Schley ND, Fu GC. J. Am. Chem. Soc. 2014; 136:16588-16593. [PubMed: 25402209]

12. Capture of 4-t-butylcyclohexyl radical often proceeds with poor-to-moderate stereoselectivity. For example, see: Damm W, Giese B, Hartung J, Hasskerl T, Houk KN, Hüter O, Zipse H. J. Am. Chem. Soc. 1992; 114:4067-4079. Osby JO, Heinzman SW, Ganem B. J. Am. Chem. Soc. 1986; 108:67-72.

13. For example, see: Lin X, Sun J, Xi Y, Lin D. Organometallics. 2011; 30:3284-3292.Fu R. Ph.D. Thesis. California Institute of TechnologyPasadena, CA2014Chapter 5Gutierrez O, Tellis JC, Primer DN, Molander GA, Kozlowski MC. J. Am. Chem. Soc. 2015; 137:4896-4899. [PubMed: 25836634]

14. For examples of previous studies of the reversibility of halogen-atom transfer in nickel-catalyzed cross-couplings of unactivated alkyl halides, see: Zultanski SL, Fu GC. J. Am. Chem. Soc. 2011; 133:15362-15364. [PubMed: 21913638] (Suzuki coupling; irreversible); b) Reference 8a (Kumada coupling; reversible).

15. In preliminary studies under our standard conditions, very modest stereoselectivity was observed in cross-couplings of secondary alkyl electrophiles that are conformationally flexible.

Angew Chem Int Ed Engl. Author manuscript; available in PMC 2018 May 15. 
Previous studies:

$\mathbf{R}^{1}$<smiles>[M]C([Tl])[Tl]</smiles>

$\mathbf{R}^{2}$

mixture of stereoisomers

$$
\mathbf{X}-\mathbf{R}
$$

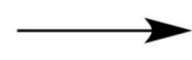

$\mathbf{R}^{1}$

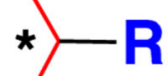

$\mathbf{R}^{2}$
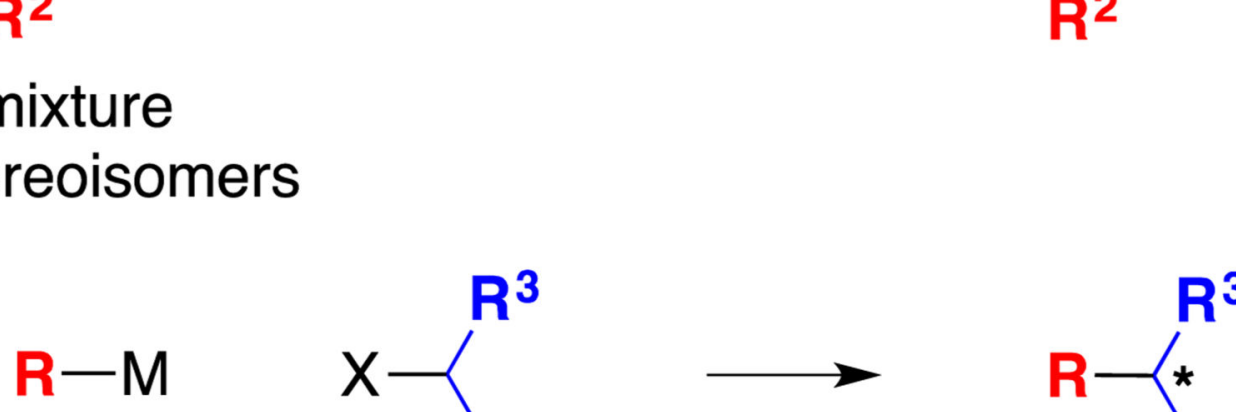<smiles>[R]C([Y])[R]</smiles>

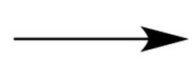

mixture of stereoisomers

This study:

$\mathbf{R}^{1}$<smiles>[M]C(C)C</smiles>

$\mathbf{R}^{2}$
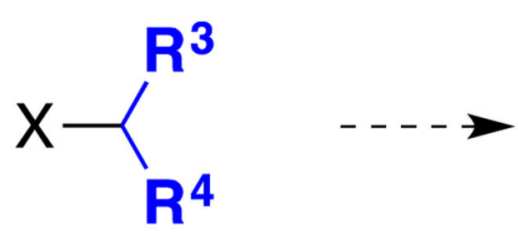

mixture of stereoisomers of stereoisomers

Figure 1.

Stereoconvergent metal-catalyzed alkyl-alkyl cross-couplings. 


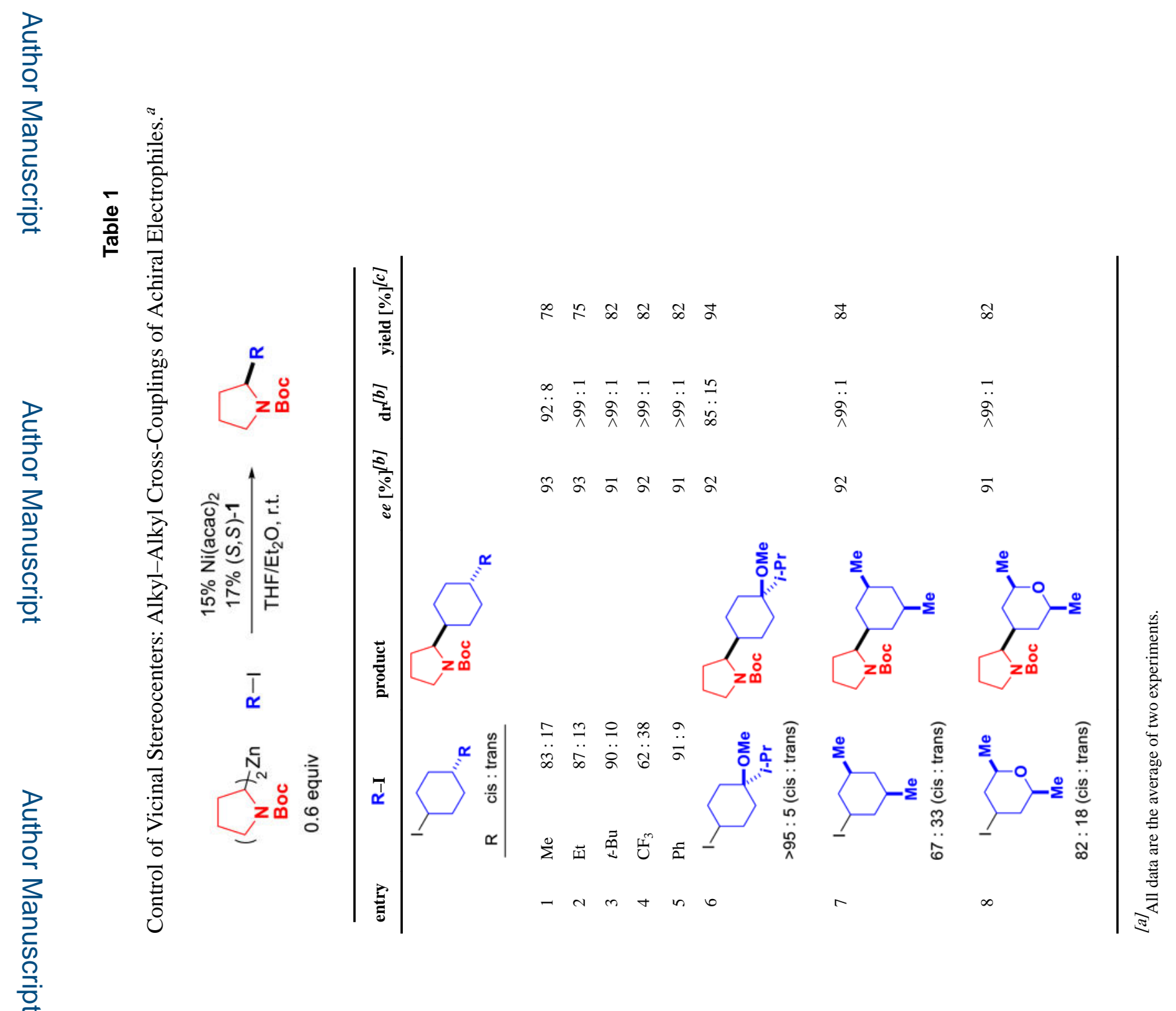



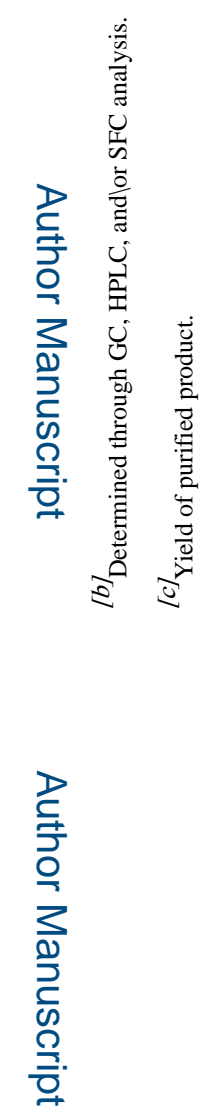

로을

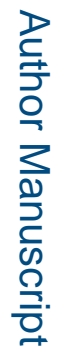

Angew Chem Int Ed Engl. Author manuscript; available in PMC 2018 May 15. 


\section{Table 2}

Control of Vicinal Stereocenters: Alkyl-Alkyl Cross-Couplings of Chiral Electrophiles. ${ }^{a}$

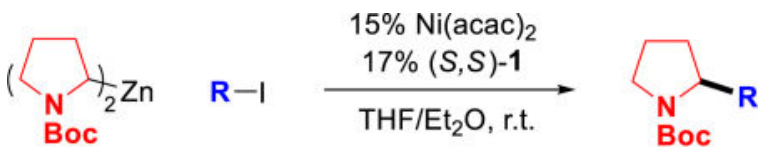

0.6 equiv

\begin{tabular}{lllll}
\hline entry & R-I & product & $\operatorname{dr}^{[b, c]}$ & yield $(\boldsymbol{\%})[\boldsymbol{c}, \boldsymbol{d}]$ \\
\hline 1 & &
\end{tabular}

2

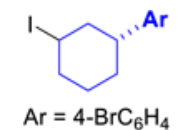

$83: 17$ (cis : trans)

3

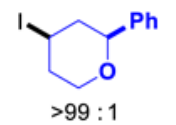

4

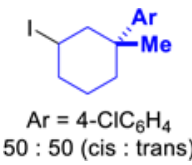

5

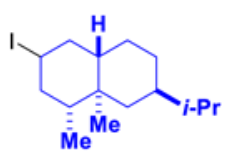

$85: 15$ (cis : trans)

6

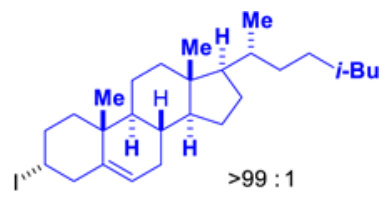

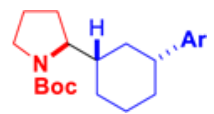

$94: 6(92: 8)$

$60(62)$

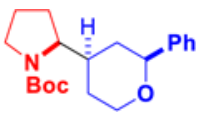

$96: 4(94: 6)$

90 (84)

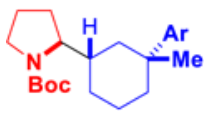

$86: 14(86: 14)$

$65(63)$

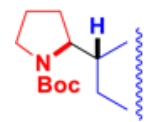

$95: 5(94: 6)$

$81(80)$

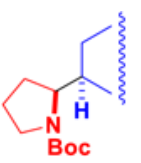

$87: 13(68: 32)$

$62(52)$

\footnotetext{
${ }^{[a]}$ The electrophilic coupling partners were $\$ 99 \%$ ee, except for entry 4 , which was $94 \%$ ee.

${ }^{[b]}$ Determined through HPLC analysis; ratio of (major stereoisomer) : (sum of all other stereoisomers).

${ }^{[c]}$ The values in parentheses are the data for $(R, R)-\mathbf{1}$.

${ }^{[d]}$ Yield of purified product.
} 